\title{
Influência da dieta na concentração sérica de triglicerídeos
}

\section{The influence of diet on the triglycerides serum concentration}

Marli Schiavo ${ }^{1}$

Adroaldo Lunardelli ${ }^{2}$

Jarbas Rodrigues de Oliveira ${ }^{3}$

\begin{abstract}
unitermos resumo
Triglicerídeos

Dia

Jejum

Introdução: Elevados níveis de triglicerídeos no soro estão associados com condições patogênicas que aceleram a aterosclerose, além de existirem evidências de que a hipertrigliceridemia é um fator de risco independente para doenças coronarianas, pois contribui para as cardiopatias devido a efeito aterogênico direto das lipoproteínas ricas em triglicerídeos. Variações muito grandes na dosagem do colesterol e triglicérides limitam sua utilização clínica. Estas variações podem ser analíticas, quando relacionadas a metodologia e a procedimentos utilizados pelos laboratórios, e pré-analíticas, quando se referem a fatores intrínsecos do indivíduo. Objetivos: Usando o mesmo método laboratorial, analisaram-se possíveis alterações nos níveis lipídicos dos pacientes a fim de questionar a verdadeira validade de o jejum de 12 horas, anterior à punção, ser suficiente para que se possam realizar dosagens fidedignas com o perfil do paciente. Material e método: Foram analisados soros de 29 pacientes, colhidos em dois distintos dias: na segunda-feira e na quinta-feira da mesma semana. Resultados: Podemos observar variações quanto à dosagem de triglicerídeos em diferentes dias da semana, quando os níveis de tal parâmetro, na segunda-feira apresentam-se mais elevados do que na quinta-feira, mesmo que o paciente tenha feito um rigoroso jejum de 12 horas antes de ambas as coletas. O colesterol total, a HDL, a LDL e a VLDL não se mostraram com variação estatisticamente significativas. Discussão: Sendo assim, o jejum recomendado de 12 horas não é suficiente para avaliar o real perfil lipídico do paciente.
\end{abstract}

Background: Elevated levels of triglycerdes in the serum are associated with patologicals conditions that they accelerate to aterosclerose, beyond they evidences will exist of that to hypertrigliceridemia is an independent factor of risk for illnesses coronarias therefore I contributed for the cardiopatias by an effect aterogênico straight of the lipoproteinas rich in triglicerídeos. Very big variations in the dosage of the colesterol and triglicérides they limit sweats clinical utilization. Those variations can be analytic, when related to metodologia and procedures utilized by the laboratories, and pré analytic, when related to factors intrínsecos of the individual. Objectives: Using the same approach laboratorial, analyzed itself possible alterations in the levels lipídicos of the patients in order to question to true validade of that the fast of 12 previous hours to the punção be sufficient for that can be carried out trustworthy dosages with the profile of the patient. Methods: They were analyzed serum of 29 patients, reaped in two distinct days; in the Monday and in the Thursday from the same week. Results: We be able to observe variations on the occasion of the dosage of triglicerideos in peculiar days from the week, being that the levels of such parameter in the Monday are presented more elevated that in the Thursday, even that the sick one I have done a rigorous fast of 12 hours before of both you collect them. The colesterol gross, the HDL, the LDL and the VLDL they were not shown with significant statistical variation. Discussion: Being like this, the fast recommended of 12 hours is not sufficient to relate the real profile lipidico of the patient.

\section{key words}

Triglycerides

Day

Fast

1. Bioquímica especialista em Análises Clínicas da Pontificia Universidade Católica do Rio Grande do Sul (PUCRS). 2. Bioquímico da PUCRS. 3. Coordenador do Laboratório de Pesquisa em Biofísica da PUCRS. Laboratório de Pesquisa em Biofísica do Departamento de Ciências Fisiológicas da Faculdade de Biociências da PUCRS. 


\section{Introdução}

Os principais lipídeos do plasma humano são colesterol, ésteres do colesteril, triglicerídeos, fosfolipídios e ácidos graxos não-esterificados. Os lipídeos são substâncias insolúveis em água. São transportados na forma de lipoproteínas, que podem ser divididas, de acordo com a sua densidade, em cinco classes: quilomícrons, lipoproteínas de densidade muito baixa (VLDL), lipoproteínas de baixa densidade (LDL), lipoproteínas de densidade intermediária (IDL) e lipoproteínas de alta densidade (HDL) (6).

A função principal das lipoproteínas plasmáticas parece ser a de transporte dos triglicerídeos e do colesterol dos locais de origem - no intestino (origem exógena) e no fígado (origem endógena) - para os locais de armazenamento e utilização (6).

Os triglicerídeos e o colesterol entram no plasma na forma de partículas de lipoproteínas ricas em triglicerídeos (quilomícrons e VLDL) e sofrem mudanças intravasculares através da enzima lipase lipoprotéica, que hidrolisa os triglicerídeos e os diglicerídeos em ácidos graxos e monoglicerídeos.

As partículas de superfície, chamadas de colesterol livre e fosfolipídeos, e as apolipoproteínas são transferidas da VLDL à HDL através da interação com a lecitinacolesterol aciltransferase (LCAT) para formar ésteres de colesteril e lisolecitina. Os ésteres do colesteril são subseqüentemente transferidos à IDL, que é convertida em LDL rica em ésteres de colesteril. A LDL é, assim, um produto final do metabolismo da VLDL intravascular $(6,17)$. O colesterol representa o principal componente da LDL, enquanto representa um componente menor das VLDL e HDL (12).

O HDL deve ser visto como tendo um papel vital no transporte do excesso de colesterol dos tecidos extra-hepáticos de volta para o fígado, por onde é excretado na bile (transporte reverso do colesterol). A remoção do excesso de colesterol celular das paredes arteriais pode ter um papel crucial em diminuir o acúmulo de colesterol nas paredes arteriais, inibindo a aterogênese (17).

Variações muito grandes na dosagem do colesterol e triglicérides limitam sua utilização clínica (13). Estas variações podem ser analíticas, quando relacionadas à metodologia e a procedimentos utilizados pelos laboratórios, e pré-analíticas quando relacionadas a fatores intrínsecos do indivíduo, estilo de vida (obesidade, idade, consumo de álcool, estresse, sedentarismo, tabagismo, entre outros), uso de medicação (drogas hipolipemientes), doenças as- sociadas (diabetes, hipotireoidismo, entre outras), procedimento de coleta e preparo da amostra (13). Os fatores pré-analíticos, especialmente os de origem biológica (intraindividual, idade, sexo e raça) são os principais responsáveis pela variabilidade dos resultados (13).

Segundo tabela de referência para a trigliceridemia desenvolvida pelo Consenso da Conferência do National Institute of Health sobre hipertrigliceridemia, valores de $500 \mathrm{mg} / \mathrm{dl}$ são considerados anormalmente altos e devem sofrer futuras avaliações e ser tratados; e valores inferiores a $150 \mathrm{mg} / \mathrm{dl}$ devem, geralmente, ser aceitos como normais. Pessoas com nível de triglicerídeos plasmáticos de jejum entre $150 \mathrm{mg} / \mathrm{dl}$ e $500 \mathrm{mg} / \mathrm{dl}$ representam um problema diferente, porque tais níveis estão associados a um risco de aproximadamente duas vezes para as doenças vasculares. Para o paciente, estes níveis de triglicerídeos podem ser normais ou representar um marcador para riscos aumentados (6). É recomendado um jejum de 12 a 14 horas antes da realização do perfil lipídico (13).

Estudos experimentais investigam a elevação dos triglicerídeos no soro associada a aumento do risco de doenças coronarianas. Surgem evidências de que a hipertrigliceridemia é um fator de risco independente para doenças coronarianas (9), pois contribui para estas doenças por um efeito aterogênico direto das lipoproteínas ricas em triglicerídeos, particularmente as VLDL (8). O tamanho das partículas de VLDL liberadas depende da disponibilidade de triglicerídeos, no fígado. As VLDL muito grandes, ricas em triglicerídeos, são secretadas quando está ocorrendo síntese excessiva de triglicerídeos hepáticos, como na obesidade, no diabetes melito não-insulino-dependente (DMNID) e no excesso de consumo de álcool. Em contrapartida, as pequenas VLDL são secretadas quando a disponibilidade de triglicerídeos, mas não a de colesterol, é diminuída (17).

Os valores de referência para o colesterol total, segundo o Expert Parol of the National Cholesterol Education Program (NCEP, 1987) são de até $200 \mathrm{mg} / \mathrm{dl}$ (17). Altos níveis de LDL e baixos níveis de HDL estão relacionados com doenças cardíacas (14). É desejável ter baixos níveis de colesterol total e LDL na circulação sangüínea, mas também é desejável ter uma concentração tão alta quanto possivel do colesterol total na forma de HDL (1).

Elevados níveis de triglicerídeos no soro estão associados com quatro condições patogênicas que aceleram a aterosclerose (11): diminuição dos níveis de HDL no soro (1); aumento das lipoproteínas remanescentes; pequena 
elevação na LDL; aumento das condições trombogênicas (9). Isto sugere que algum tipo de hipertrigliceridemia poderá ser a causa de aterosclerose (9). O aumento da $\mathrm{HDL}$ retarda o aparecimento de aterosclerose e conseqüentemente faz a diminuição da progressão das doenças da artéria coronária. A HDL exerce efeito protetor com enzimas antioxidantes que podem prevenir algum processo inflamatório inicial (8). O papel antiaterogênico da HDL tem implicações importantes para o controle das doenças cardíacas (8).

Embora a síntese acentuada de triglicerídeos possa levar a um aumento da eliminação de triglicerídeos, o número de partículas de VLDL liberadas não está necessariamente aumentando. Ao contrário, são liberadas partículas maiores de VLDL contendo mais triglicerídeos (17). A elevação rápida dos triglicerídeos reflete um incremento nas VLDL. A origem da confusão é que nem todas as VLDL são aterogênicas e a determinação laboratorial dos níveis de triglicerídeos não distingue entre aterogênicos (pequenas VLDL) e não-aterogênicos (partículas grandes de VLDL) (3).

O aumento dos triglicerídeos está associado ao risco de doenças cardiovasculares, com um risco maior nas muIheres que nos homens $(2,15)$, porém, depois do ajustamento do HDL-colesterol e de outros fatores, estes riscos diminuíram significativamente (10). Os triglicerídeos são um fator de risco independente para doenças cardiovasculares e há urgente necessidade de baixar os níveis de triglicerídeos no plasma, diminuindo assim o risco de doenças cardíacas (2).

A hipercolesterolemia é a causa direta da aterosclerose, enquanto a hipergliceridemia é mais bem vista como um marcador para o aumento do risco de doenças arteriocoronarianas (5).

O NCEP recomenda que, além da análise das histórias clínicas pessoal e familiar, as dosagens de colesterol total, HDL, LDL e triglicerídeos sejam realizadas de cinco em cinco anos para a identificação de indivíduos com risco elevado de desenvolver uma doença arterial crônica (DAC) em indivíduos com mais de 20 anos (10).

O presente trabalho tem como objetivo avaliar os níveis de colesterol total, HDL, LDL, VLDL e triglicerídeos em diferentes dias da semana nos mesmos pacientes. Usando-se o mesmo método laboratorial, analisar-se-ão possíveis alterações nos níveis lipídicos a fim de se questionar a verdadeira validade de o jejum de 12 horas anterior à punção ser suficiente para que se possam realizar dosagens fidedignas com o perfil do paciente.

\section{Materiais e métodos}

Foram analisados soros de 29 pacientes, colhidos por punção venosa em dois dias distintos: na segunda-feira e na quinta-feira da mesma semana. As duas coletas foram feitas nos mesmos indivíduos. Os pacientes foram orientados a realizar jejum prévio de 12 horas.

Os indivíduos selecionados foram pacientes ambulatoriais, homens $(n=17)$ e mulheres $(n=12)$ sem censura de idade (entre 37 e 68 anos) do município de Campos Borges (RS). As colheitas e as dosagens se procederam no mês de julho de 2002. As análises foram feitas com a distinção dos dias da semana e também com a diferenciação do sexo dos pacientes. Fez-se ainda uma análise de correlação entre a idade e os níveis de triglicerídeos.

Foram dosados triglicerídeos, colesterol total e HDL-colesterol por método enzimático colorimétrico (Labtest Diagnostica) com equipamento semi-automático (Labquest - Labtest Diagnostica). Os triglicérides foram mensurados a partir de uma quinineimina formada pela ação de enzimas como a lipase lipoprotéica. O HDL-colesterol foi determinado no sobrenadante após o tratamento da amostra com ácido fosfofungístico e cloreto de magnésio, que precipitam seletiva e quantitativamente as VLDL e LDL. A dosagem de colesterol se dá pela mensuração de uma quinidina formada pelos ésteres de colesterol com a ajuda da colesterol estearase, da colesterol oxidase e da peroxidase.

Por meio da equação de Friedwald puderam-se mensurar os níveis de LDL-colesterol e VLDL-colesterol somente nos pacientes que apresentaram triglicerídeos com níveis inferiores a $400 \mathrm{mg} / \mathrm{dl}$.

Através dos resultados obtidos nas análises foram calculados as médias, o desvio padrão e a significância (Oneway Anova) com análise de Bonferroni.

\section{Resultados}

A dosagem de triglicerídeos na segunda-feira mostrase mais elevada que a mensuração dos mesmos na quinta-feira, como mostra a Figura 1. Há uma diferença estatisticamente significativa que mostra a queda dos níveis de triglicérides, elucidando alteração nos níveis plasmáticos nos pacientes quando em dias diferentes.

A análise da concentração sérica do colesterol total mostrou que este parâmetro não sofre oscilação perante os diferentes dias da semana analisados. Como elucida a Figura 2, não há diferenças estatisticamente significativas entre os grupos. 


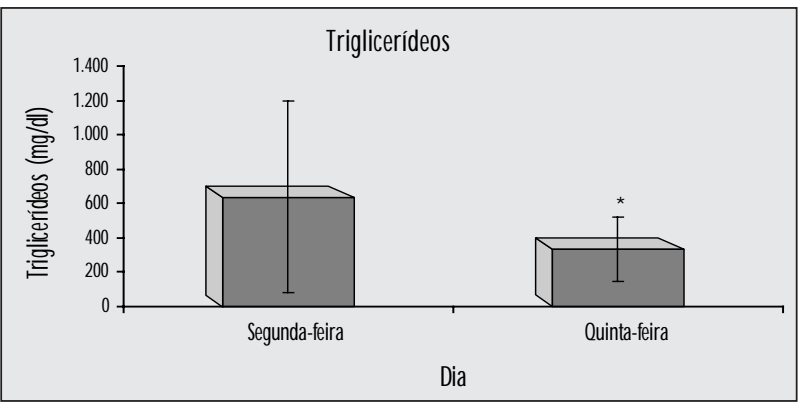

Figura 1 - Média e desvio padrão da concentração de triglicerídeos encontrada no soro dos pacientes em diferentes dias da semana. As diferenças estatisticamente significativas $(p<0,05)$ entre os dois grupos estão indicadas por *

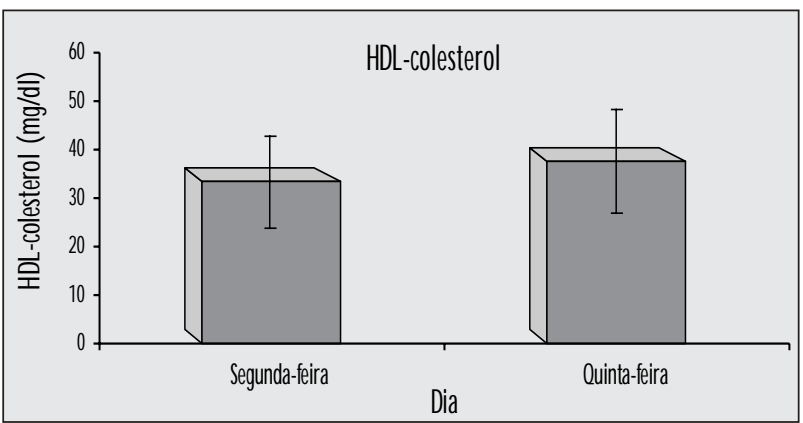

Figura 3 - Média e desvio padrão da concentração de HDL-colesterol encontrada no soro dos pacientes em diferentes dias da semana

Os resultados obtidos referentes à lipoproteína de alta densidade (Figura 3) não mostram alteração significativas em seus níveis.

As análises referentes à lipoproteína de baixa densidade (Figura 4) e à de muito baixa densidade (Figura 5) apresentaram-se sem alterações significativas. Sendo assim, ambos os parâmetros mencionados - LDL e VLDL não apresentam diferenças estatísticas significativas quando da dosagem em distintos dias da semana.

As análises dos lípides (triglicerídeos, HDL e colesterol total) com a diferenciação por sexo mostraram diferença significativa somente na observação do parâmetro triglicerídeos (Figura 6). Somente nos pacientes do sexo masculino há uma diminuição significativa dos triglicérides entre os dias da semana. Nota-se ainda que, tanto na segunda-feira quanto na quinta-feira, os níveis de triglicerídeos das mulheres apresentam-se em níveis significativamente mais baixos.

Uma análise de correlação serve para determinar se dois conjuntos de dados se relacionam, isto é, se os maiores valores de um conjunto estão associados com os maiores valores do outro (correlação positiva), se os menores valores de um conjunto estão associados com os maiores do outro (correlação negativa), ou se os valores

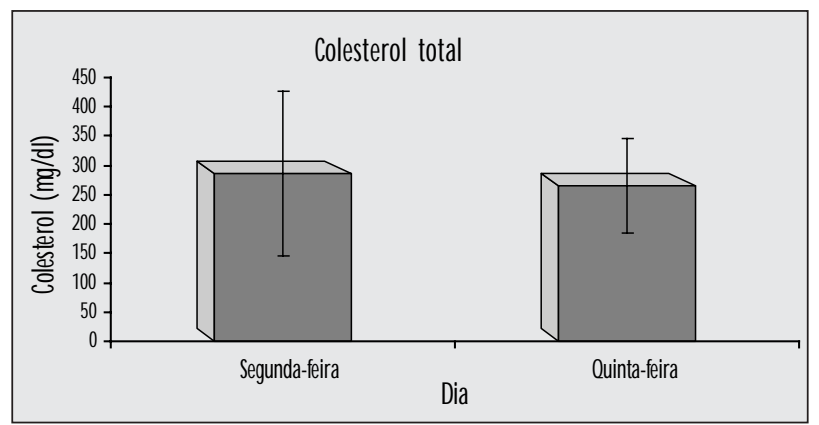

Figura 2 - Média e desvio padrão da concentração de colesterol total encontrada no soro dos pacientes em diferentes dias da semana

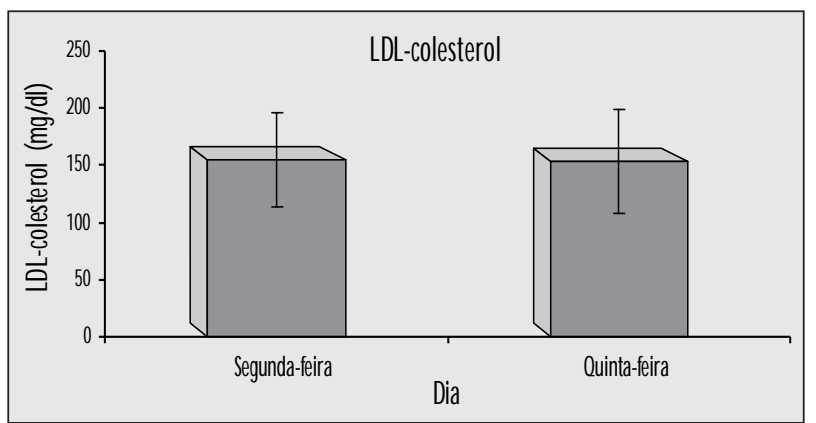

Figura 4 - Média e desvio padrão da concentração de LDL-colesterol encontrada no soro dos pacientes em diferentes dias da semana

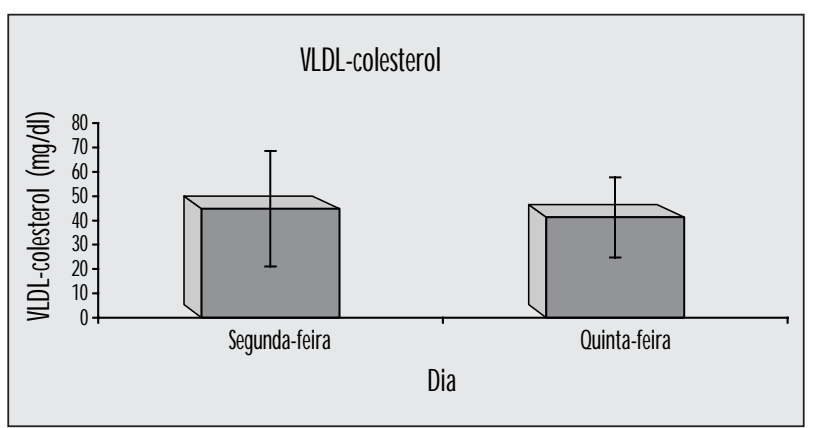

Figura 5 - Média e desvio padrão da concentração de VLDL-colesterol encontrada no soro dos pacientes em diferentes dias da semana

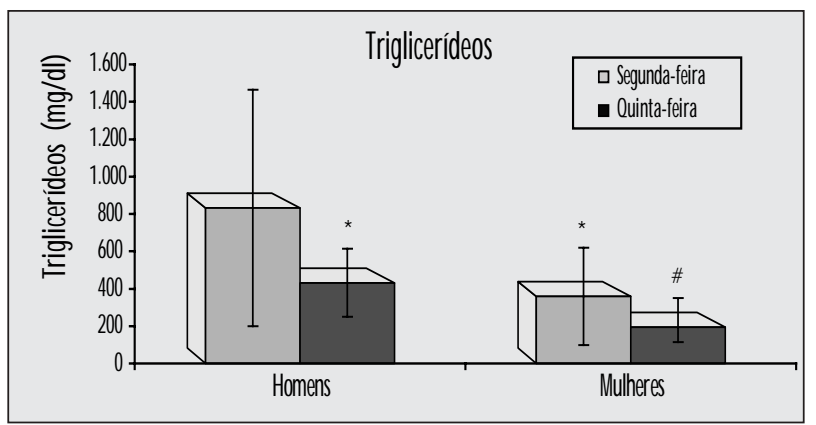

Figura 6 - Média e desvio padrão da concentração de triglicerídeos encontrada no soro dos pacientes em diferentes dias da semana. As diferenças estatisticamente significativas $(p<0,05)$ em relação ao grupo de homens da segunda-feira estão indicadas por *. As diferenças estatisticamente significativas $(p<0,05)$ em relação ao grupo de homens da quinta-feira estão indicadas por \# 
dos dois conjuntos não se relacionam (correlação próxima a zero).

A análise de correlação entre a diferença da concentração sérica de triglicerídeos (na segunda-feira e na quinta-feira) e a idade $\left(\mathrm{R}^{2}=0,17\right)$ permite-nos concluir haver correlação próxima a zero (Figura 7), ou seja, há pouca correlação entre os dois parâmetros.

\section{Discussão}

O aumento dos triglicerídeos é um distúrbio lipídico relativamente freqüente na prática clínica. Os níveis pósprandiais de triglicerídeos aumentam dentro de duas horas (faixa de duas a dez horas) após a ingestão de alimentos. Recomenda-se, portanto, jejum de 12 a 16 horas antes da coleta da amostra, pois pode haver uma variação de $25 \%$ a $50 \%$ (faixa de $18 \%$ a $100 \%$ ) entre os dias. Esta grande variação de um dia para outro está bem observada nos níveis dosados de triglicerídeos (12).

A obesidade, o estresse agudo, a ingestão de álcool, as dietas com alto teor de gordura e doces, a gravidez, a estrogenoterapia, a terapia de glicocorticóides e um número considerado de doenças (diabetes, pancreatite aguda, síndrome nefrótica, gota e uremia, entre outros) aumentam consideravelmente os níveis de triglicerídeos (12).

Os triglicerídeos têm um papel primário na formação de aterosclerose em se tratando de hipertrigliceridemia (11). A hipertrigliceridemia é o distúrbio lipídico de mais fácil controle, pois os níveis de triglicérides podem ser satisfatoriamente controlados por mudanças nos hábitos de vida, dieta equilibrada, aumento da atividade física e restrição ao álcool. Raramente encontra-se hipertrigliceridemia isolada, sendo freqüentemente acompanhada por aumento no colesterol total, no LDL-colesterol e diminuição no HDL-colesterol.

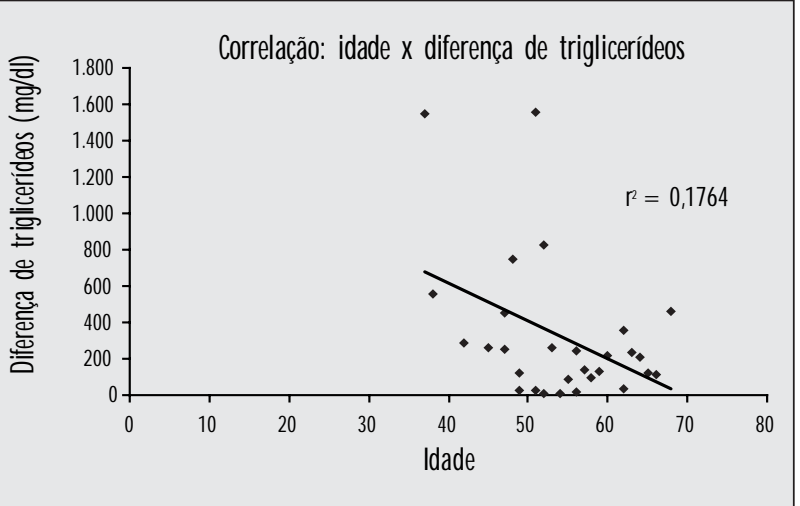

Figura 7 - Correlação entre a diferença dos níveis de triglicerídeos (segunda e quinta-feira) e a idade dos pacientes
Em nosso estudo, podemos observar variações estatisticamente significativas ocorridas quanto às dosagens de triglicerídeos em diferentes dias da semana, quando os níveis de tal parâmetro na segunda-feira se apresentam mais elevados que na quinta-feira, mesmo que o paciente tenha feito um rigoroso jejum de 12 horas antes de ambas as coletas. Tal diferença ocorre, também, quando se analisam pacientes do sexo masculino. Este aumento dos triglicerídeos na segunda-feira se deve ao fato de que, tradicional e incondicionadamente, a alimentação mantida pela população no fim de semana difere das refeições sustentadas nos dias úteis, já que na primeira há uma predileção por alimentos mais gordurosos e de alto valor lipídico, principalmente pelos pacientes do sexo masculino. Constata-se, portanto, que o jejum de 12 horas não é suficiente para tornar a análise do parâmetro de triglicerídeos fidedigna com as reais condições do paciente, já que os níveis de tal lípide se mostram suscetíveis à ingesta de alimentos 12 horas antes da colheita da amostra.

Os triglicérides mostram-se mais elevados nos homens do que nas mulheres em ambos os dias da semana (segunda e quinta-feira), como elucidado em nosso estudo. Segundo estudos prévios, os níveis de triglicerídeos são menores nas mulheres (7), corroborando nosso trabalho.

Não há correlação entre a idade e a queda da concentração de triglicerídeos. A pequena correlação mostra não haver qualquer influência da idade dos pacientes na diminuição dos triglicérides na quinta-feira.

O HDL-colesterol não se mostrou com variação estatística significativa, porém ocorreu um discreto aumento em seus níveis. Existe uma relação aproximadamente inversa entre os níveis de triglicerídeos e de $\mathrm{HDL}$, de modo que elevados níveis de triglicerídeos tendem a estar associados a baixos níveis de HDL (12).

Notou-se que, em relação ao colesterol total, as variações foram muito pequenas e não-significativas estatisticamente, podendo-se dizer que o colesterol não acompanha a queda dos triglicérides, sendo estes dois parâmentros independentes.

Podemos concluir com este estudo, portanto, que os níveis séricos de triglicérides são sujeitos a flutuações entre os diferentes dias da semana, já que dependem do teor alimentar. Sendo assim, o jejum recomendado de 12 horas não é suficiente para relatar o real perfil lipídico do paciente. A avaliação dos lipídios deve sempre levar em conta fatores ambientais e individuais, por isso o paciente não deve ser medicado sem uma adequada interpretação 
de sua situação, devendo, se necessário, realizar novo exame em um dia da semana que esteja na sua rotina, sem excessos de alimentação e bebidas alcoólicas. A dosagem de triglicerídeos na segunda-feira se torna deficitária por- que leva a interpretações errôneas, além de causar erros, também, na dosagem do HDL-colesterol, já que níveis altos de triglicerídeos causam diminuição deste e, conseqüentemente, um aumento dos níveis de LDL-colesterol.

\section{Referências}

1. Abbasi, F. et al. High carbohydrate diets, triglyceride-rich lipoproteins, and coronary heart disease risk. Am J Cardiol., 85: 45-8, 2000.

2.A ustin, M.A.;H okanson,J.E. \& Edwards, K.L. H ypertriglyceridemia as a cardiovascular risk factor. Am J Cardiol., 81(4A): 7B12B, 1998.

3. Cullen, P. Evidence that triglycerides are an independent coronary heart disease risk factor. Am J Cardiol., 86: 9439, 2000.

4. Franceschini, G. Epidemiologic evidence for high-density lipoprotein cho lesterol as a risk factor for coronary artery disease. Am J Cardiol., 88: 9N -13N , 2001.

5. Grundy, S.M. H ypertriglyceridemia, atherogenic dyslipidemia, and the metabolic syndrome. Am J Cardiol., 81(4A): 18B-25B, 1998.

6. Henry,J.B. Diagnósticos clínicos e tratamento por métodos laboratoriais. 18 ed. São Paulo: Manole, 1998.

7. Lennep, J.E.R. et al. Risk factors for coronary heart disease: implications of gender. Cardiovascular Research., 53: 53849, 2002

8. Libby, P. Managing the risk of atherosclerosis: the role of highdensity lipoprotein. Am J Cardiol., 88: 3N -8N , 2001.
9. N akaya, N . Hyper triglyceridemia as a cause of atherosclerosis. Nippon Rinsho., 60(5): 860-7, 2002.

10. NIH. Consensus D evelopment Panel on Triglyceride, HighD ensity Lipoprotein, and Coronary H eart D isease. JAM A, 269(4): 505-10, 1993

11. Rapp, R.J. Hypertriglyceridemia: a review beyond low-density lipoprotein. Cardiology in Review, 10(3): 163-72, 2002.

12. Ravel, R. Laboratório clínico: aplicações clínicas dos dados laboratoriais. 6 ed. Rio de Janeiro: Guanabara Koogan, 1997.

13. Recomendações para o exame do perfil lipídico para clínicos e laboratórios. Atheros., 10(4): 109-20, 1999.

14. Sacks, F.M. The relative role of low-density lipoprotein cholesterol and high-density lipoprotein cholesterol in coronary artery disease: evidence from large-scale statin and fibrate trials. Am J Cardiol., 88(12A): 14N -8N , 2001.

15. Sposito, A.C .et al.Triglyceride and lipoprotein (a) are markers of coronary artery disease severity among postmeno pausal women. Maturitas, 39: 203-8, 2001.

16. Sprecher, D.L.Triglycerides as a risk factor for coronary artery disease. Am.J. Cardiol., 82: 49U -56U, 1998.

17.W yngaarden, J.B. \& Smith, L.H. Cecil Tratado de M edicina Interna. 18 ed. Rio de Janeiro: Guanabara Koogan, 1990. 